\title{
Starting laminar plumes: Comparison of laboratory and numerical modeling
}

\section{Judith Vatteville}

Institut de Physique du Globe, UMR 7579, CNRS, 4 place Jussieu, F-75252 Paris CEDEX 05, France

(jvattevi@ipgp.jussieu.fr)

Also at Laboratoire FAST, UMR 7609, Université Pierre et Marie Curie, Université Paris-Sud XI, CNRS, F-91405

Orsay, France

\section{Peter E. van Keken}

Department of Geological Sciences, University of Michigan, Ann Arbor, Michigan 48109-1005, USA (keken@umich.edu)

\section{Angela Limare}

Institut de Physique du Globe, UMR 7579, CNRS, 4 place Jussieu, F-75252 Paris CEDEX 05, France (limare@ipgp.jussieu.fr)

\section{Anne Davaille}

Laboratoire FAST, UMR 7609, Université Pierre et Marie Curie, Université Paris-Sud XI, CNRS, F-91405 Orsay, France (davaille@fast.u-psud.fr)

[1] A detailed comparison of starting laminar plumes in viscous fluids is provided using the complementary approaches of laboratory modeling and numerical simulation. In the laboratory experiments the plumes are started in a nearly isoviscous silicone oil with heat supplied through a fixed circular source. The temperature field is measured by differential interferometry and thermochromic liquid crystals. The velocity field is determined by particle image velocimetry. Numerical simulations of the laboratory experiments are performed using a finite element method that employs the measured properties of the physical oil and the heating history. No further adjustments are made to match the laboratory results. For fluids at two different viscosities and for variable power supplied to the plume there is excellent agreement in the temporal evolution and fine spatial detail of the plume. Minor differences remain, particulary in the transient stage of the plume in the low-viscosity fluid, but the differences are within the experimental uncertainties. In contrast, the assumption of constant viscosity in the numerical models leads to differences that are larger than the experimental uncertainties, demonstrating that these near-isoviscous fluids should not be considered to have constant viscosity.

Components: 4865 words, 7 figures, 2 tables.

Keywords: mantle plumes; simulation; validation; verification.

Index Terms: 0550 Computational Geophysics: Model verification and validation; 0560 Computational Geophysics: Numerical solutions (4255); 8121 Tectonophysics: Dynamics: convection currents, and mantle plumes.

Received 14 July 2009; Revised 23 October 2009; Accepted 29 October 2009; Published 22 December 2009. 


\section{Introduction}

[2] The precise determination of the characteristics of viscous plumes starting from a hot source is important for a better understanding of the dynamics of mantle plumes and their suggested support of hot spot regions [Morgan, 1971; Davies, 1999; Schubert et al., 2001; Ito and van Keken, 2007]. Fluid dynamical models of plumes have been developed using a variety of approaches, including theoretical models [Batchelor, 1954; Worster, 1986], laboratory experiments [Shlien, 1976; Griffiths, 1986; Campbell and Griffiths, 1990; Moses et al., 1993; Kaminski and Jaupart, 2003; Coulliette and Loper, 1995; Davaille and Vatteville, 2005; Whitehead and Luther, 1975; Griffiths and Campbell, 1991; Kerr and Mériaux, 2004; Whittaker and Lister, 2006], and numerical models [Olson et al., 1993; van Keken and Gable, 1995; Davies, 1995; Farnetani and Richards, 1995; Albers and Christensen, 1996; Kellogg and King, 1997].

[3] While in some cases similarity of scaling laws has been shown between laboratory results and predictions from theory [e.g., Kaminski and Jaupart, 2003], the differences in model setup, boundary conditions and heating modes make a detailed comparison generally difficult. In only rare cases direct numerical simulation of laboratory plume experiments has been provided [van Keken, 1997]. The strength of the laboratory experiments lies in the direct verification of fluid dynamics using analogue materials, but the measurement of temperature and velocity fields is indirect. The numerical techniques solve directly for the temperature, velocity and pressure, but discretization issues and choices in the solution techniques can potentially cause inaccurate or incorrect predictions. It is therefore useful to directly compare laboratory and numerical techniques for a given fluid dynamical experiment.

[4] In this technical brief we will focus on a detailed intercomparison of numerical and laboratory models of starting laminar plumes in a nearly isoviscous fluid at high Prandtl number. This direct comparison of laboratory studies and numerical simulations provides essential validation and verification of independent approaches. It also provides a means to estimate the magnitude of the errors introduced by each technique and by the assumptions made in the modeling.

\section{Experimental and Numerical Setup}

[5] We use as fluid the silicone oils Rhodorsil 47 V500 and the ten times more viscous
47V5000. These fluids have nearly constant properties over the temperature range considered in the experiments. The fluid fills a tank with a rectangular base of $150 \times 150 \mathrm{~mm}^{2}$ to a height $H=$ $158 \mathrm{~mm}$ above the heater assemblage. The oils are mixed with thermochromic liquid crystals. The properties of the fluids are provided in Table 1. These properties were all measured at IPGP and FAST except for the heat capacity for which we used the manufacturer's value. Special care was taken to measure the viscosity law, which can different from the manufacturer's values by $5 \%$ to $10 \%$ and depends on the fluid batch and its age. We use for the numerical simulations the viscosity that was determined from rheometry done at the same time as the laboratory experiments on the same batch of fluids (Figure 2a).

[6] The heater assemblage that is placed in the center of the tank consists of a small rectangular Peltier device below a slightly larger and circular copper plate with a diameter of $18 \mathrm{~mm}$. When a voltage difference is put across the Peltier device, it heats the top and cools the bottom. The Peltier device rests on an aluminum plate which causes rapid diffusion of the cold away from the heater assemblage. The high conductivity of the copper $(400 \mathrm{~W} / \mathrm{mK}$, compared to $0.17-0.18 \mathrm{~W} / \mathrm{mK}$ for the fluid) causes a uniformly hot patch. A separate set of thermocouples monitor the air temperature around the tank and the temperature of the fluid far away from the heater.

[7] The tank is illuminated by a 2-D laser sheet (see Figure 1) that is centered over the heating patch. Digital images are taken of the illuminated section at a frequency between 0.5 and $3 \mathrm{~Hz}$. The laser illumination allows for the determination of the fluid flow and temperature characteristics without perturbing the flow.

[8] The temperature field is measured by the independent techniques of thermochromic liquid crystals (TLC) and differential interferometery (DI). These two techniques provide fully independent measurements of the temperature of the fluid in the section illuminated by the laser. The first technique uses the property that TLCs are opaque over a narrow temperature range and therefore show up as narrow contour lines when illuminated by a laser (Figure 2b). For silicone oils the TLC bandwidth is between $2^{\circ} \mathrm{C}$ and $3^{\circ} \mathrm{C}$ (Figure $2 b$ ), which is significantly larger than that for aqueous solutions. The TLCs provide therefore less accurate quantitative predictions in the silicone oils. The DI uses interference of light phase distortions due to temperature 
Table 1. Properties of the Silicone Oils ${ }^{\mathrm{a}}$

\begin{tabular}{lccccrr}
\hline \multicolumn{1}{c}{ Fluid } & $\rho\left(\mathrm{kg} / \mathrm{m}^{3}\right)$ & $\alpha(1 / \mathrm{K})$ & $\eta(\mathrm{Pa} \mathrm{s})$ & $k(\mathrm{~W} / \mathrm{mK})$ & $C_{p}(\mathrm{~J} / \mathrm{kgK})$ & $\operatorname{Pr}$ \\
\hline 47V500 & 991 & $9.4 \times 10^{-4}$ & $\exp \left(b_{0}+b_{1} / T_{k}\right)$ & 0.17 & 1460 & $4.5 \times 10^{3}$ \\
47V5000 & 991 & $9.4 \times 10^{-4}$ & $\exp \left(b_{2}+b_{3} / T_{k}\right)$ & 0.18 & 1460 & $4.5 \times 10^{4}$ \\
\hline
\end{tabular}

${ }^{a}$ The values for thermal expansivity $\alpha$, dynamic viscosity $\eta$, and conductivity $k$ are measured at $20^{\circ} \mathrm{C}$. Density is measured across the experimental temperature range, and the provided value is at $0^{\circ} \mathrm{C}$. The heat capacity $C_{p}$ is provided by the vendor. In the dynamic viscosity law the temperature $T_{k}$ is absolute temperature in Kelvin. The coefficients determined by a best fit to the measured viscosity over a $40^{\circ}$ range are $b_{0}=$ $-7.11, b_{1}=1892, b_{2}=-3.66$, and $b_{3}=1550$. The measured viscosity for both fluids is about $10 \%$ lower than the viscosity description provided by the manufacturer but has similar temperature dependence (of a factor of 2 over a $40^{\circ} \mathrm{C}$ range). We have measured the viscosity of the $47 \mathrm{~V} 500$ fluid three different times on two different rheometers (a ThermoRheo RS600 at IPGP and an Anton Paar MCR501 at FAST) and found that the measured values were within $5 \%$ of each other. This is a consequence of the inherent uncertainties in rheometry. While this error is quite small, it should be taken into account when interpreting a comparison like this where differences between measured quantities are on the same order.

gradients. In these experiments we obtain a precision of about $2 \%$ and provide therefore highly accurate predictions of the temperature in the plume.

[9] The velocity field is measured by Particle Image Velocimetry (PIV) which uses sequential cross correlation between two consecutive images. We use the PIV package DaVis from LaVision, with a square interrogation window of 32 pixels and overlap of $50 \%$, leading to a spatial resolution of $3 \mathrm{~mm}$ and a precision of $\sim 5 \%$. For a full description of the imaging techniques see Davaille and Limare [2007] and Limare et al. [2008].

[10] We simulate the laboratory experiments numerically by the discretization of the governing equations using a finite element method. We use the properties of the fluid as shown in Table 1. We

a)

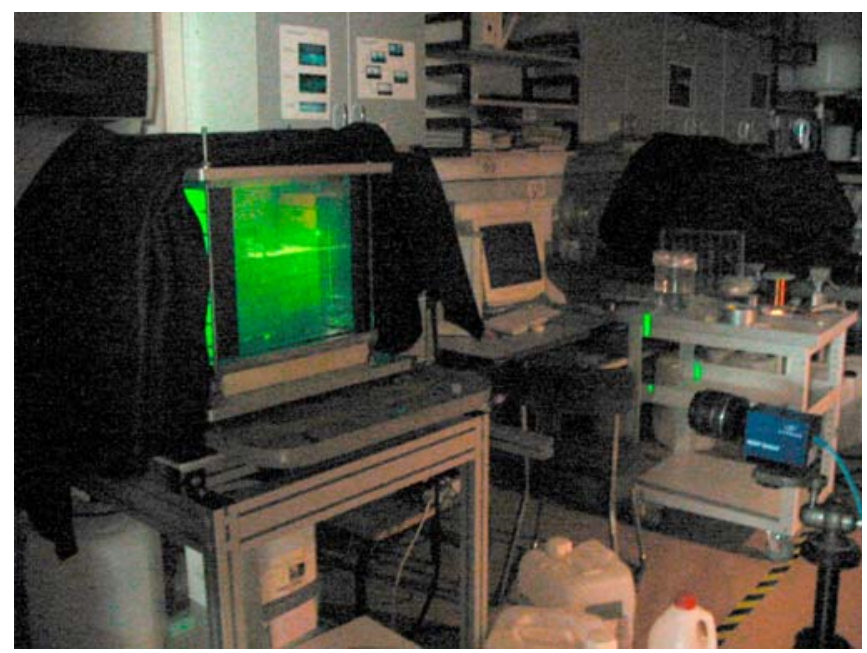

assume that the plume is axisymmetric and use an axisymmetric cylindrical geometry with a radius of $8.5 \mathrm{~cm}$. This provides a geometry for the numerical setup that has the same cross-sectional area as that in the laboratory.

[11] The governing equations are those of the conservation of mass, conservation of momentum, and conservation of energy, in an incompressible and infinite Prandtl number fluid:

$$
\nabla \cdot v=0
$$

$$
\begin{gathered}
\nabla P=\nabla \cdot \boldsymbol{\sigma}+\rho g \\
\rho C_{p}\left[\frac{\partial T}{\partial t}+(v \cdot \nabla) T\right]=\nabla(k \nabla T)
\end{gathered}
$$

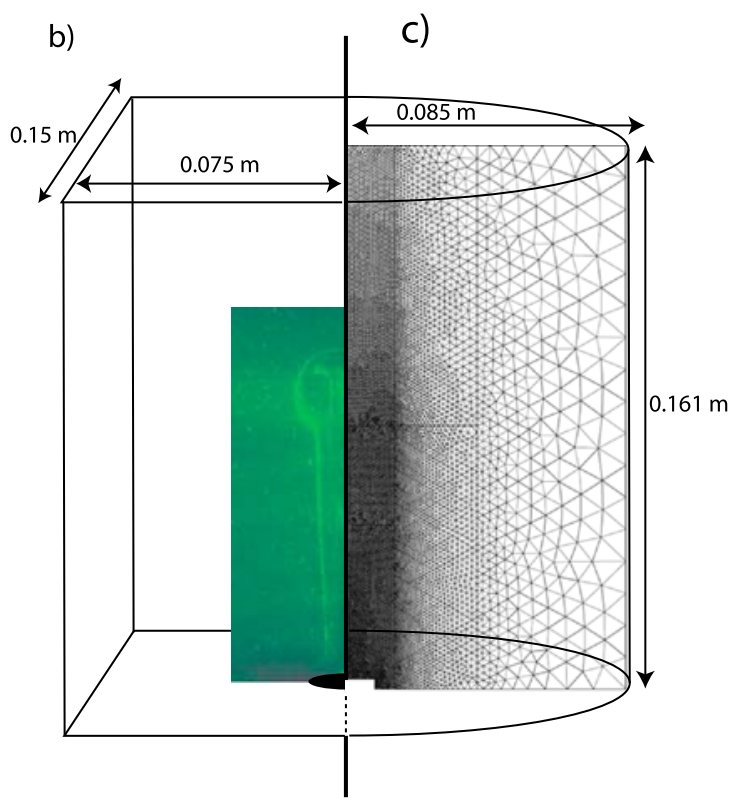

Figure 1. (a) Photo of the laboratory setup (at IPGP) demonstrating the laser sheet (in green), heater assemblage (minor bright point at base of tank), and digital camera. (b) Illustration of the tank geometry and (c) the axisymmetric finite element model. The green area in Figure $1 \mathrm{~b}$ shows a typical photograph of a plume with temperature contours illuminated by the TLC, and the quadratic elements of the finite element mesh which has a resolution of $0.2 \mathrm{~mm}$ near the heater are shown in Figure 1c. 


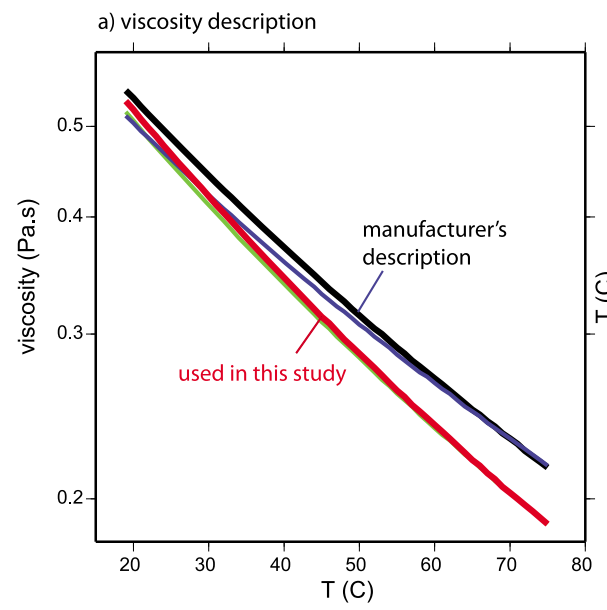

b) TLC intensity in $47 \mathrm{~V} 5000$

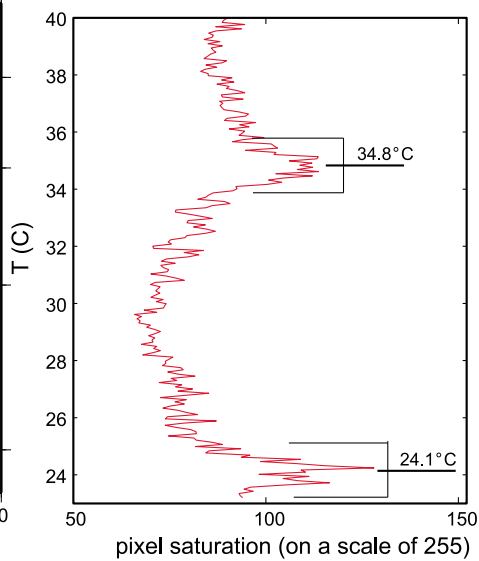

c) temperature distribution in heater

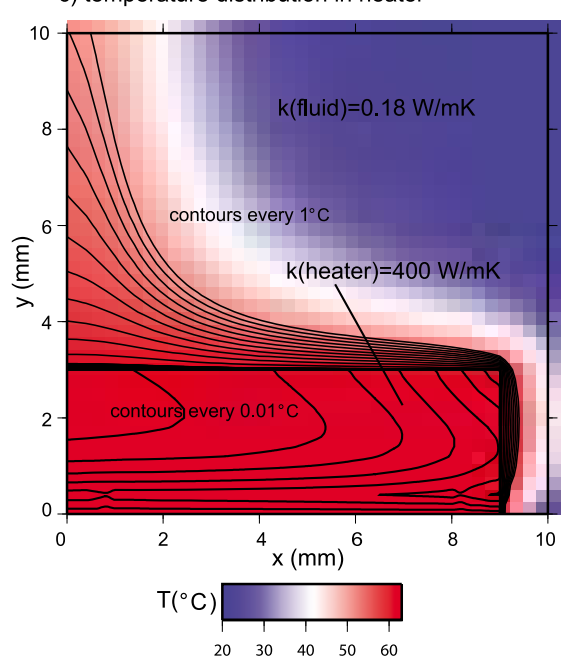

Figure 2. (a) Viscosity measurements for the fluid 47V500. The black line shows the viscosity formulation provided by the manufacturer. The other lines show the three different interpolations of measurements made during a 2 year period at the labs at IPGP and FAST. The red line shows the formulation used in the numerical modeling. (b) TLC calibration: the temperature is shown as a function of light intensity (measured as pixel saturation on an 8 bit scale). For silicone oils the peaks have a bandwidth of $2^{\circ}-3^{\circ}$. (c) Simulation of the heating of the copper plate, demonstrating that the high conductivity of the copper results in a nearly uniform temperature distribution.

where $\boldsymbol{v}$ is velocity in the fluid, $P$ is pressure, $\boldsymbol{\sigma}$ is the stress tensor $\boldsymbol{\sigma}=\eta\left(\nabla \boldsymbol{v}+\nabla \boldsymbol{v}^{T}\right), \eta$ is the viscosity of the fluid, $\rho$ is the density, $g$ the gravitational acceleration, $C_{p}$ the specific heat of the fluid, $T$ the temperature and $k$ the conductivity. We use SI units throughout, except for temperature which is in degrees Celsius unless otherwise specified. We take into account the slight temperature dependence of the viscosity (Table 1).

[12] The boundary conditions are chosen to mimic those of the laboratory tank. We use no-slip conditions on the bottom and side boundaries and free slip at the top boundary. The side and top boundaries are kept at room temperature. The bottom boundary is also at room temperature, except at the heater, where we prescribe the measured timedependent evolution of the temperature in the heater. The heater temperature time series are provided as auxiliary material. ${ }^{1}$ The use of this temperature boundary condition at the heater is justified due to the high conductivity of the copper plate. We have verified this assertion in a series of experiments where we measured the temperature at two different places in the copper plate. We found the same values to within the thermocouple precision $\left(0.05^{\circ} \mathrm{C}\right)$. We also simulated explicitly the temperature distribution using finite element mod-

\footnotetext{
${ }^{1}$ Auxiliary materials are available at ftp://ftp.agu.org/apend/gc/ 2009gc002739. Other auxiliary material files are in the HTML.
}

eling and found similarly that the temperature differences are less than $0.1^{\circ} \mathrm{C}$ (Figure 2c).

[13] We use the Sepran finite element method [Cuvelier et al., 1986] with a penalty function method for the Stokes equation and streamline upwinding for the heat equation. We use a grid spacing $0.2 \mathrm{~mm}$ near the heater and along the lower part of the plume axis, with decreasing resolution toward the top and side boundaries (Figure 1).

\section{Results}

[14] In Figure 3 we compare the laboratory measurements of temperature using DI (horizontal lines) and TLC (image in the background on the left of each frame) with the numerical simulation (contour lines on right) for a typical experiment with fluid 47V5000. An animation of the laboratory experiment is available in the auxiliary material. The TLCs show up over two temperature ranges as the darker greys in the background. The ranges over which the TLCs are opaque are $33.3^{\circ} \mathrm{C}-36.3^{\circ} \mathrm{C}$ and $23.1{ }^{\circ} \mathrm{C}-25.1{ }^{\circ} \mathrm{C}$ (Figure 2b). The positions where DI measures the same temperature ranges at specific heights in the fluid are shown by the horizontal lines. The effects of diffusion are particularly well displayed in the widening of the cooler region over which the TLCs are opaque and clearly cover the same band as that predicted by the numerical model. The comparison shows 

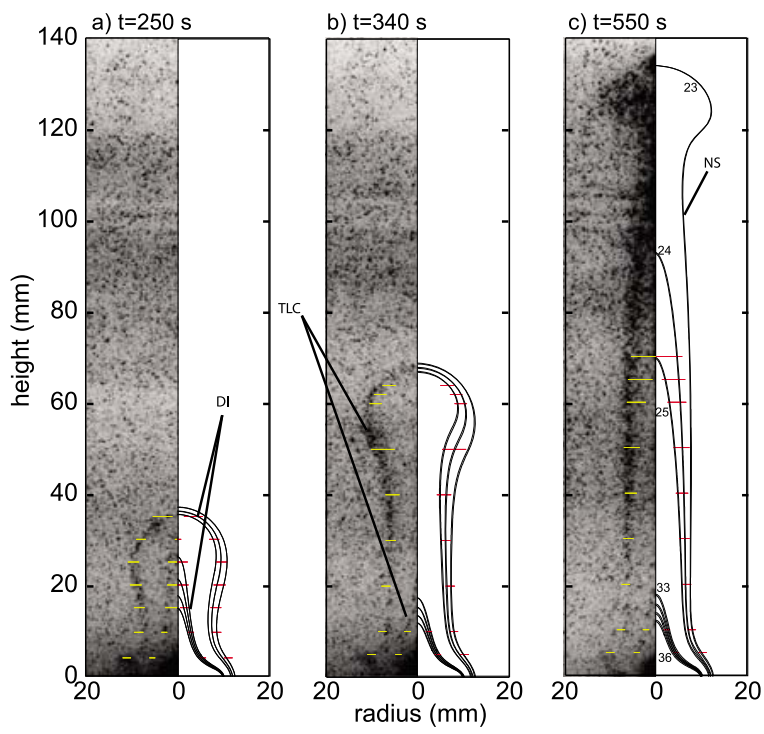

Figure 3. Temperature evolution of a plume in fluid $47 \mathrm{~V} 5000$ at electrical power $P=1.17 \mathrm{~W}$. Each of the snapshots shows on the left of the image the thermochromic liquid crystals (TLC) in inverted intensity scale (so that the bright lines in Figure 1b show up as dark regions). The TLC are opaque in the intervals $23.1^{\circ} \mathrm{C}-$ $25.1{ }^{\circ} \mathrm{C}$ and $33.3^{\circ} \mathrm{C}-36.3^{\circ} \mathrm{C}$. The grey scale image is overlain by the same temperature range as measured by the differential interferometry (DI) with yellow indicating the range for the TLCs. On the right of each image the temperature field predicted by the numerical method (NS) is shown using $1{ }^{\circ} \mathrm{C}$ contours in the intervals $33^{\circ} \mathrm{C}-36^{\circ} \mathrm{C}$ and $23^{\circ} \mathrm{C}-25^{\circ} \mathrm{C}$. The DI results are shown in red on the right for these same intervals.

very good agreement between the two approaches and suggests that the independent laboratory measurements can reproduce the temperature field to well within $1^{\circ} \mathrm{C}$ precision.

[15] The PIV allows for a direct determination of the velocity field in the plane of the laser sheet. We will show the results of a number of simulations at various powers in fluid 47V500. The characteristics of the six different simulations are provided in Table 2. Table 2 shows that as expected, the temperature difference, the viscosity contrast, and the thermal Rayleigh number all increase with increasing power. We provide two different definitions of the Rayleigh number. The first is based on the radius of the heater $R=9 \mathrm{~mm}$, which is a length scale close to the plume radius [Griffiths, 1986; Kerr and Mériaux, 2004]:

$$
R a_{1}=\frac{\rho g \alpha \Delta T R^{3}}{\kappa \eta}
$$

where $\kappa=k / \rho c_{p}$ and $\Delta T$ is the difference between the steady state heater temperature and room temperature. The second definition uses the height of the fluid above the heater $H=158 \mathrm{~mm}$ :

$$
R a_{2}=\frac{\rho g \alpha \Delta T H^{3}}{\kappa \eta}
$$

This second definition comes from the classical case of Rayleigh-Bénard convection in which the whole lower surface is at constant temperature. A value $R a_{2}>10^{6}$ indicates that the box is much higher than the thickness of the hot thermal boundary layer and that plume-shaped instabilities can develop [Davaille and Limare, 2007]. It is important to point out that the dynamics of the plumes does not change much if the height of the box is increased.

[16] A snapshot of a typical simulation at power $\mathrm{P}=1.0 \mathrm{~W}$ is shown in Figure 4a. The measured velocity field (Figure 4b) is slightly noisy due to the statistical nature of PIV, but compares qualitatively and quantitatively well with the velocity field predicted by the numerical method (Figure 4a). We observe that the near-steady plume conduit velocity predicted by the numerical method is consistently higher than the laboratory measurements. One critical aspect of the laboratory measurements is that the PIV method uses an averaging window which is necessary to compile statistically meaningful velocities. For these experiments we used an averaging window of $3 \mathrm{~mm}$ wide and $3 \mathrm{~mm}$ high. As a consequence the PIV method will always underestimate the conduit velocity. Due to the

Table 2. Characteristics of the Model Simulations in Fluid 47V500

\begin{tabular}{lllllll}
\hline & \multicolumn{7}{c}{ Power P (W) } \\
\cline { 2 - 7 } & \multicolumn{1}{c}{0.6} & \multicolumn{1}{c}{1.0} & \multicolumn{1}{c}{1.3} & 1.7 & 2.3 & \multicolumn{1}{c}{3.3} \\
\hline Room T $\left({ }^{\circ} \mathrm{C}\right)$ & 21.47 & 19.56 & 20.79 & 19.69 & 21.47 & 20.63 \\
Maximum T $\left({ }^{\circ} \mathrm{C}\right)$ & 42.4 & 45.8 & 52.5 & 56.2 & 64.5 & 74.2 \\
Viscosity at room T (Pa s) & 0.504 & 0.526 & 0.512 & 0.524 & 0.504 & 0.513 \\
Viscosity at maximum T (Pa s) & 0.329 & 0.309 & 0.273 & 0.256 & 0.222 & 0.190 \\
$R a_{1}$ (based on heater dimension) & $2.27 \times 10^{3}$ & $2.13 \times 10^{3}$ & $3.38 \times 10^{3}$ & $3.8 \times 10^{3}$ & $4.66 \times 10^{3}$ & $5.70 \times 10^{3}$ \\
$R a_{2}$ (based on height of box) & $1.23 \times 10^{7}$ & $1.47 \times 10^{7}$ & $1.83 \times 10^{7}$ & $2.06 \times 10^{7}$ & $2.52 \times 10^{7}$ & $3.08 \times 10^{7}$ \\
\hline
\end{tabular}



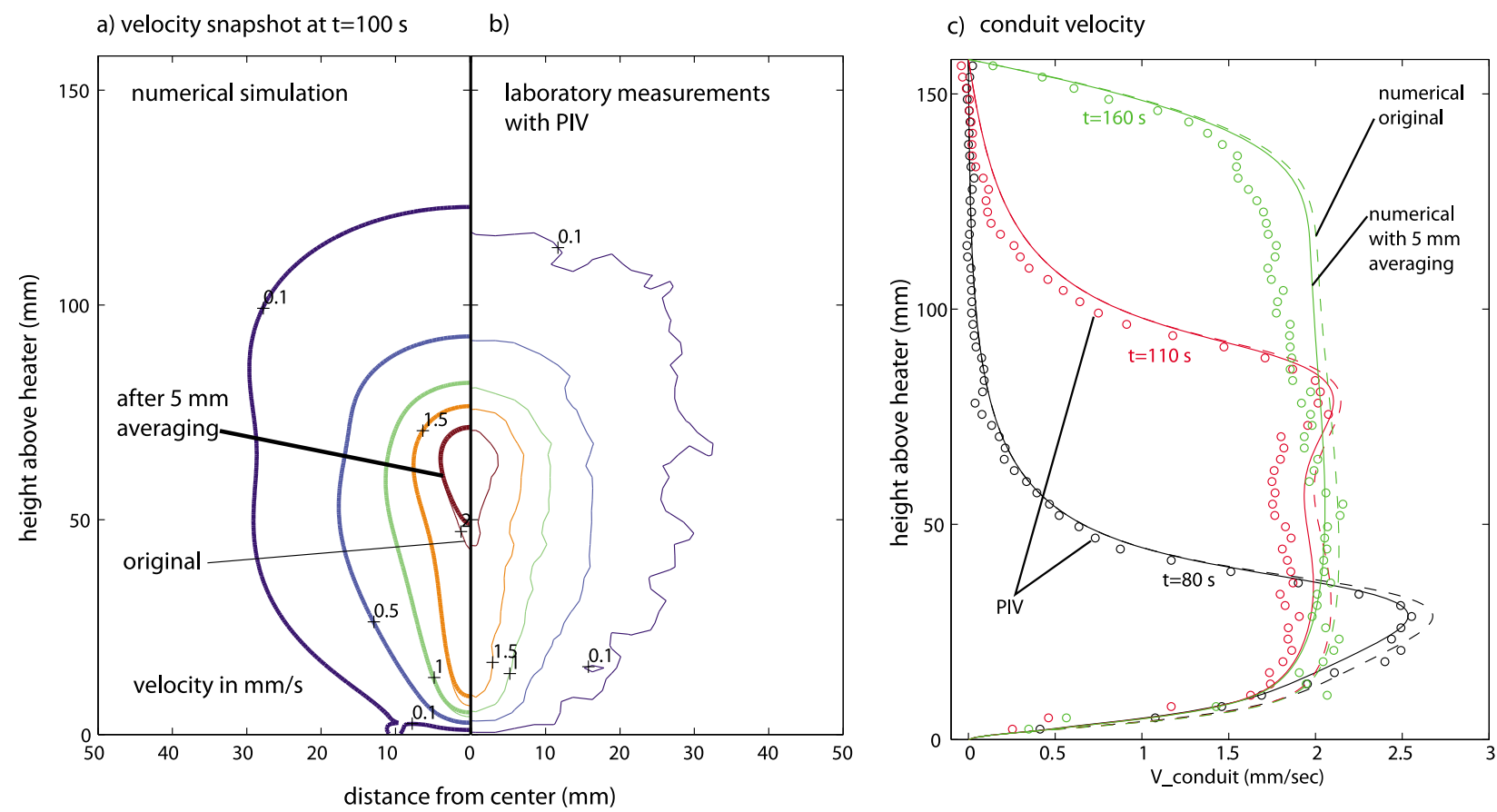

Figure 4. Comparison of velocity fields determined in the lab and predicted by the numerical techniques for an experiment in fluid 47V500 with an electrical heater power of $1.0 \mathrm{~W}$. Comparison of the 2-D velocity structure at time $100 \mathrm{~s}$ showing the PIV determined velocity from (a) the laboratory experiment and (b) the numerical predictions. (c) Plot of the velocity along the conduit for times $t=80,110$, and $160 \mathrm{~s}$. PIV results are shown as open circles. The numerical predictions are shown with dashed lines for the original full velocity and with solid lines after averaging with a $5 \mathrm{~mm}$ by $3 \mathrm{~mm}$ window.

difficulty of alignment of the averaging window with the center of the plume, the horizontal averaging could be weighted somewhat more strongly to the slower velocities in the boundary layers. To mimic the effects of averaging we employed a 3 to $5 \mathrm{~mm}$ averaging width in the numerical simulations.

[17] The vertical cross section of the velocity fields (Figures $4 \mathrm{a}$ and $4 \mathrm{~b}$ ) shows that the effect of averaging is most dominant near the center of the plume. The use of similar averaging windows provides a very good agreement between the laboratory and numerical simulations, especially for the maximum value of the axis velocity and within the foot and the head of the plume. These are the regions where the temperature gradients are strongest. This strongly suggests that optical distortion due to the temperature dependence of the fluid refraction index has a negligible effect on the velocity measurements.

[18] From the PIV and the numerical simulation we extract the velocity profile along the center axis of the plume (Figure 4c). We note the excellent agreement between the two approaches in the fine details of the stem velocity near the heater for these three snapshots. We observe minor systematic shifts between the laboratory measurements and numerical prediction in the top half of the plume conduit (Figure 4c). At this stage we do not understand the reasons for this discrepancy. We think it is unlikely that a more viscous skin developed since the fluids were protected from dust during the experiments and the oils do not contain solvents, suggesting that drying effects should be minimal. We suspect that the discrepancy is caused by differences in boundary conditions between laboratory (rectangular box with minimum distance between boundaries and plume center of $7.5 \mathrm{~cm}$ ) and numerical simulation (axisymmetric cylinder with constant distance of $8.5 \mathrm{~cm}$ ). This would suggest a somewhat more pronounced slowing in the laboratory setup of the plume in the later stages, as is observed.

[19] To demonstrate that the differences are not due to resolution issues in the numerical simulation, we have performed a divergence test (which is the same as a convergence test, except cheaper) by decreasing the mesh resolution for a number of cases (Figure 5). In this case we notice only very 


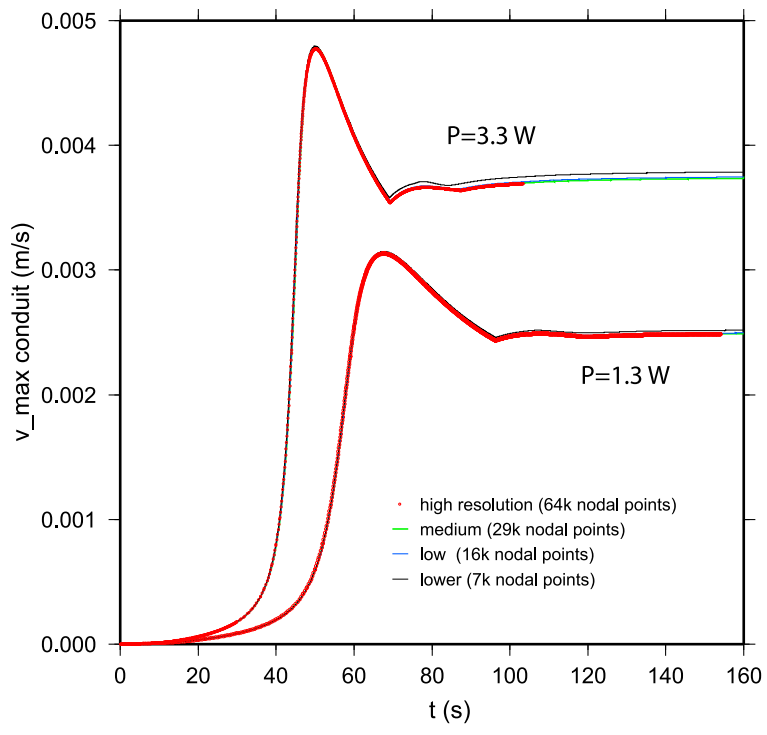

Figure 5. Resolution test for the numerical simulations for fluid 47V500 and powers $\mathrm{P}=1.3$ and $3.3 \mathrm{~W}$. The red dots show the results on the high-resolution mesh (with 63,683 nodal points) that was used for the numerical simulations shown in Figures 5 and 6. The green and blue curves show the results for intermediate and low resolution. The results only deviate noticeably for the higher-power model when ten times fewer nodal points are used (black curve).

a) Temperature in heater

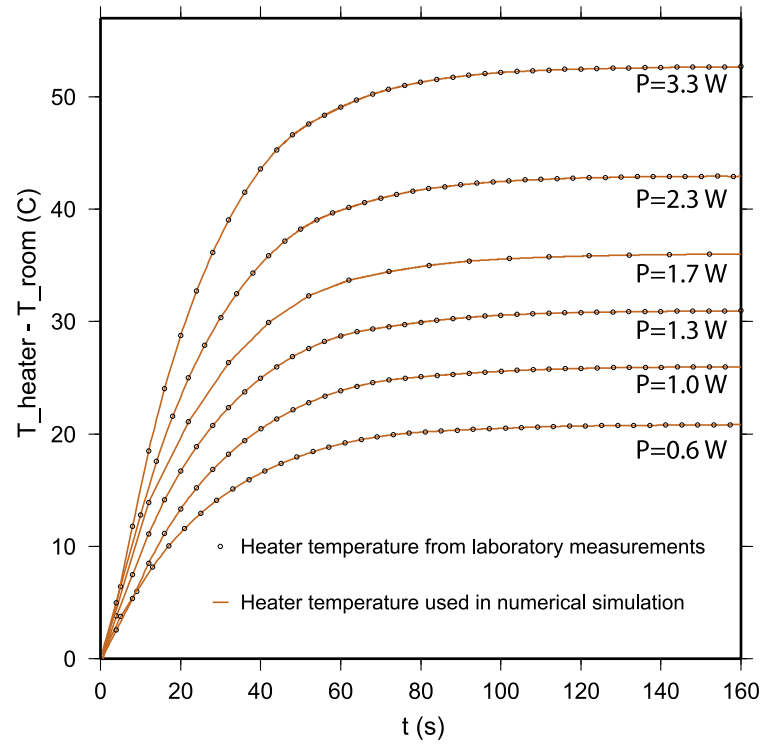

small differences when the number of elements has been reduced by a factor of 4 . It is only when the number of elements is nearly 10 times lower that a more significant offset is observed for the highpower case.

[20] The two previous cases are for laboratory experiments at a specific electrical power $P$ that is supplied to the Peltier device. We demonstrate in Figure 6 that the laboratory and numerical techniques show good agreement over a range of supplied powers (and hence over a range of temperature of the heater assemblage). In this case we plot the maximum velocity $V_{\max }$ along the plume conduit as a function of time. The solid line shows the predicted conduit velocity maximum from the numerical simulation. As in Figure 4 we use a 3 to $5 \mathrm{~mm}$ averaging window which leads to the lower velocity shown by the small red and yellow symbols in Figure 6 . The velocity is very low during the early conductive growth [Whitehead and Luther, 1975], shows a rising peak as the plume head rises, after which the conduit velocity reaches a steady state. The $5 \mathrm{~mm}$ averaging provides excellent agreement for the final stem velocity. The discrepancy seen with the slightly smaller averaging window increases with increasing temperature, which reflects the stronger viscosity reduction in the conduit and the resulting narrower b) Maximum velocity in the center of the plume

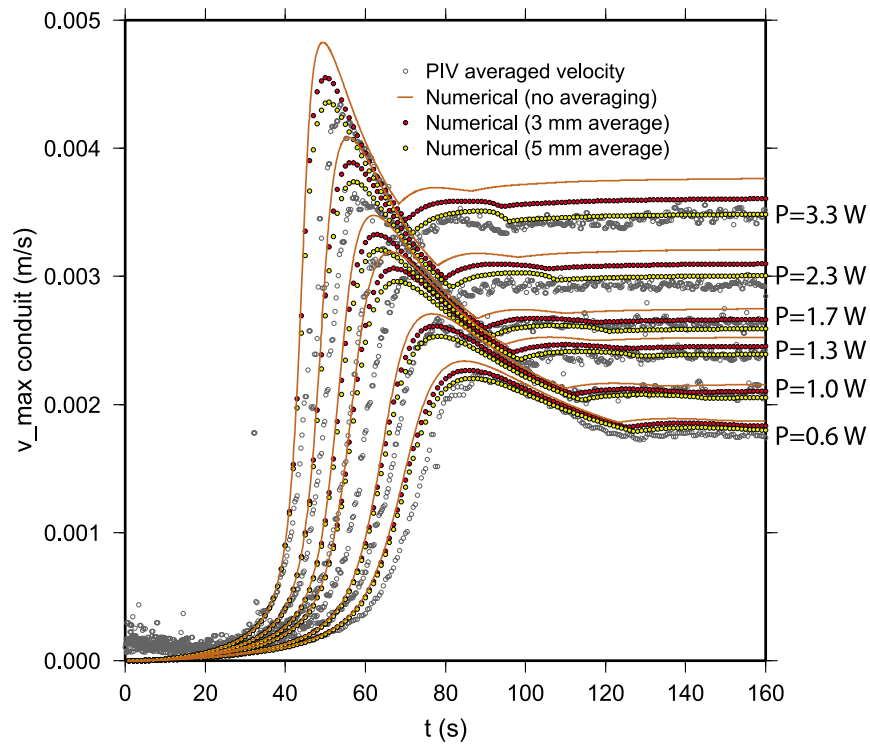

Figure 6. (a) Difference between heater and room temperature as a function of time for the six experiments in fluid 47V500. (b) Comparison of the laboratory measurements (open dots) and numerical predictions for the maximum velocity in the conduit for a series of experiments in 47V500 with variable power. The numerical prediction for the velocity at the centerline is shown in solid lines. The colored dots show the numerical results that are averaged, similar to the PIV measurements, using an averaging window of $3 \mathrm{~mm}$ by $3 \mathrm{~mm}$ (in red) and $5 \mathrm{~mm}$ by $3 \mathrm{~mm}$ (in yellow). 


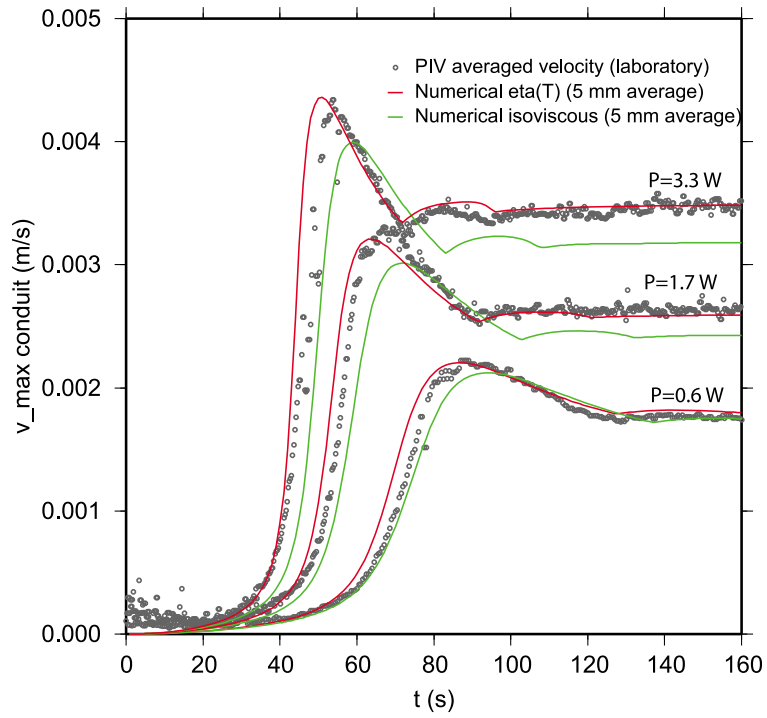

Figure 7. Comparison of conduit velocity (with a $5 \mathrm{~mm}$ by $3 \mathrm{~mm}$ averaging window) using the numerical simulation for various powers and the measured rheology (solid red lines, same as in Figure 6b) and those for a strictly isoviscous rheology (solid green lines).

velocity peak. The conductive growth stage is indicated early with the zero numerical line. The PIV data are plotted as magnitudes. While they are on average about zero they show a positive magnitude that effectively indicates the noise level of the PIV method for the static fluid.

[21] The numerical results show a consistent albeit small shift of the build up of the head and a higher peak velocity. We note that the differences are generally on the same order as the expected errors in the PIV method.

[22] The viscosity dependence of the fluid is small (Figure 2a) but not insignificant. To demonstrate the error that is incurred by the assumption of constant viscosity over this moderate temperature range we perform a few numerical simulations using the constant viscosity of the fluid at room temperature (Figure 7). As is expected, the differences increase with increasing power and temperature. There is a significant mismatch in the final steady state stem velocity and the development of the plume is delayed. The errors induced by the assumption of constant viscosity are significantly larger $(10 \%)$ than the experimental uncertainties.

\section{Summary and Discussion}

[23] In this technical brief we have demonstrated that numerical techniques can be successfully used to reproduce laboratory experiments of starting laminar plumes. The direct comparison of the indirectly measured temperature and velocity with the numerical predictions shows very good to excellent agreement for two fluids across a wide range of heater power.

[24] The comparison showed that the PIV averaging leads to a moderate but not insignificant underprediction of the conduit velocity in the laboratory measurements, suggesting that laboratory models that use PIV should be interpreted with some caution. On the positive side, the comparison demonstrates that optical distortion is negligible in the interpretation of PIV.

[25] There remain a few consistent albeit minor differences between the numerical and laboratory simulations in the transient stage. We also observe a stronger reduction in the plume velocity in the top half of the plume in the laboratory models. At this stage we do not know specifically what is causing these differences, but there are a number of possibilities that we can point out: (1) the thermal expansivity and conductivity are somewhat temperature-dependent $(1 \%-2 \%$ over the temperature range considered), but are assumed constant in the numerical simulations; (2) the heater assembly has a simplified representation compared to that in the laboratory since only the circular copper plate is modeled; (3) the origin time of the experiments could be still uncertain by 1 or $2 \mathrm{~s}$; and (4) the boundary conditions are slightly different between the numerical and laboratory setups.

[26] The good agreement between the two approaches suggests that even for these weakly temperature-dependent viscosity fluids, it is not accurate to assume that the fluids are isoviscous. This comparison also demonstrates that the infinite Pr assumption used in the numerical models is valid for these high Pr fluids within this experimental setup. This is apparently at odds with same predictions that suggest a strong dependence of the plume velocity at finite $\operatorname{Pr}$ [Worster, 1986; Kaminski and Jaupart, 2003]. We will show elsewhere that this apparent discrepancy is resolved by taking into account the differences in boundary conditions between these studies.

[27] We provide the temperature evolution of the heater for the various experiments in the auxiliary material. We hope this will stimulate other researchers to use these experiments for model validation and verification and that perhaps this may become part of a benchmark for plume 
modeling, similar to the benchmark efforts of Blankenbach et al. [1989], van Keken et al. [1997, 2008], and King et al. [2009].

\section{Acknowledgments}

[28] We thank C. Lithgow-Bertelloni and R. C. Kerr for very helpful and constructive reviews. This research has been supported by the French DyETI program from INSU/CNRS and ANR "BeGDY" and the U.S. National Science Foundation (EAR-IF 0651056 and EAR-CSEDI 0855487).

\section{References}

Albers, M., and U. R. Christensen (1996), The excess temperature of plumes rising from the core-mantle boundary, Geophys. Res. Lett., 23(24), 3567-3570.

Batchelor, G. K. (1954), Heat convection and buoyancy effects in fluids, Q. J. R. Meteorol. Soc., 80, 339-358.

Blankenbach, B., et al. (1989), A benchmark comparison for mantle convection codes, Geophys. J. Int., 98, 23-38.

Campbell, I. H., and R. W. Griffiths (1990), Implications of mantle plume structure for the evolution of flood basalts, Earth. Planet. Sci. Lett., 99, 79-93, doi:10.1016/0012821X(90)90072-6.

Coulliette, D. L., and D. E. Loper (1995), Experimental, numerical and analytical models of mantle starting plumes, Earth. Planet. Sci. Lett., 92, 143-167.

Cuvelier, C., A. Segal, and A. A. van Steenhoven (1986), Finite Element Methods and Navier-Stokes Equations, D. Reidel, Dordrecht, Netherlands.

Davaille, A., and A. Limare (2007), Laboratory studies in mantle convection, in Treatise on Geophysics, vol. 7, Mantle Dynamics, edited by D. Bercovici, pp. 89-166, Elsevier, Amsterdam.

Davaille, A., and J. Vatteville (2005), On the transient nature of mantle plumes, Geophys. Res. Lett., 32, L14309, doi:10.1029/2005GL023029.

Davies, G. F. (1995), Penetration of plates and plumes through the mantle transition zone, Earth. Planet. Sci. Lett., 133, 507-516, doi:10.1016/0012-821X(95)00039-F.

Davies, G. F. (1999), Dynamic Earth: Plates, Plumes and Mantle Convection, 458 pp. Cambridge Univ. Press, Cambridge, U. K.

Farnetani, C. G., and M. A. Richards (1995), Thermal entrainment and melting in mantle plumes, Earth. Planet. Sci. Lett., $136,251-267$.

Griffiths, R. W. (1986), Thermals in extremely viscous fluids, including the effect of temperature-dependent viscosity, J. Fluid Mech., 166, 115-138.

Griffiths, R. W., and I. H. Campbell (1991), On the dynamics of long-lived plume conduits in the convecting mantle, Earth Planet. Sci. Lett., 103, 214-227.

Ito, G., and P. E. van Keken (2007), Hotspots and melting anomalies, in Treatise on Geophysics, vol. 7, Mantle Dynamics, edited by D. Bercovici, pp. 371-436, Elsevier, Amsterdam.
Kaminski, E., and C. Jaupart (2003), Laminar starting plumes in high Prandtl-number fluids, J. Fluid Mech., 478, 287298.

Kellogg, L. H., and S. D. King (1997), The effect of temperature dependent viscosity on the structure of new plumes in the mantle: Results of a finite element model in a spherical, axisymmetric shell, Earth. Planet. Sci. Lett., 148, 13-26, doi:10.1016/S0012-821X(97)00025-3.

Kerr, R. C., and C. Mériaux (2004), Structure and dynamics of sheared mantle plumes, Geochem. Geophys. Geosyst., 5, Q12009, doi:10.1029/2004GC000749.

King, S. D., C. Lee, P. E. van Keken, W. Leng, S. Zhong, E. Tan, N. Tosi, and M. C. Kameyama (2009), A community benchmark for 2D Cartesian compressible convection, Geophys. J. Int, in press.

Limare, A., I. Kumagai, J. Vatteville, and A. Davaille (2008), Thermal plumes visualisation: Differential interferometry versus thermochromic liquid crystals, paper presented at the 13th International Symposium on Flow Visualization, Univ. of Franche-Comté, Nice, France, 1 -4 July . (Available at http://www.ipgp.fr/limare/318.pdf)

Morgan, W. J. (1971), Convection plumes in the lower mantle, Nature, 230, 42-43.

Moses, E., G. Zocchi, and A. Libchaber (1993), An experimental study of laminar plumes, J. Fluid Mech., 251, 581601.

Olson, P., G. Schubert, and C. Anderson (1993), Structure of axisymmetric mantle plumes, J. Geophys. Res., 98, 68296844.

Schubert, G., D. L. Turcotte, and P. Olson (2001), Mantle Convection in the Earth and Planets, 940 pp. Cambridge Univ. Press, Cambridge, U. K.

Shlien, D. J. (1976), Some laminar thermal and plume experiments, Phys. Fluids, 19, 1089-1098.

van Keken, P. E. (1997), Evolution of starting mantle plumes: A comparison between numerical and laboratory models, Earth. Planet. Sci. Lett., 148, 1-11.

van Keken, P. E., and C. W. Gable (1995), The interaction of a plume with a rheological boundary: A comparison between two- and three-dimensional models, J. Geophys. Res., 100, 20,291-20,302.

van Keken, P. E., S. D. King, H. Schmeling, U. R. Christensen, D. Neumeister, and M.-P. Doin (1997), A comparison of methods for the modeling of thermochemical convection, J. Geophys. Res., 102, 22,477-22,495.

van Keken, P. E., et al. (2008), A community benchmark for subduction zone modeling, Phys. Earth Planet. Inter., 171, $187-197$.

Whitehead, J. A., and D. S. Luther (1975), Dynamics of laboratory diapir and plume models, J. Geophys. Res., 80, $705-717$.

Whittaker, R. J., and J. R. Lister (2006), Steady axisymmetric creeping plumes above a planar body, part 2. A distributed source, J. Fluid Mech., 567, 379-397.

Worster, M. G. (1986), The axisymmetric laminar plume: Asymptotic solution for large Prandtl number, Stud. Appl. Math., 75, 139-152. 